\title{
The impact of the Lucentis, Eylea, Avastin in Vein Occlusion (LEAVO) Study in Australia
}

\author{
Samantha Fraser-Bell $\mathbb{1}^{1,2} \cdot$ Mark C. Gillies ${ }^{1,2}$
}

Received: 18 October 2019 / Accepted: 22 October 2019 / Published online: 4 December 2019

(c) The Royal College of Ophthalmologists 2019

Just 12 years ago, an evidence-based systematic review of treatments for central retinal vein occlusion (CRVO) reported that there was no level 1 evidence of any intervention improving visual outcomes [1]. The landscape has changed greatly since then. Many randomised controlled studies of different vascular endothelial derived growth (VEGF) inhibitors and corticosteroids have shown that these intravitreal treatments can improve vision very effectively. Most would agree that VEGF inhibitors are now considered first-line agents in the management of macular oedema due to CRVO. But, as clinicians, how do we decide which VEGF inhibitor to use? As well as differing costs to the patient and health care providers, there may be differing clinical efficacies. The Lucentis, Eylea, Avastin in Vein Occlusion (LEAVO) study was originally designed to answer whether bevacizumab or aflibercept were noninferior to ranibizumab, which was the only drug licenced to treat CRVO in the UK when it was designed. Aflibercept became licensed in the UK for CRVO after the protocol was finalised so a post hoc analysis was included to also answer whether bevacizumab was noninferior to aflibercept [2].

The LEAVO study was a well-designed, randomised clinical trial of 463 eyes with cystoid macular oedema due to CRVO enroled in 44 NHS sites. The primary endpoint was change in best corrected visual acuity measured in LogMAR letters from baseline to 100 weeks in the study eye of patients. The predefined margin for testing noninferiority between the various agents was -5 letters.

Reassuringly the LEAVO study found that all three agents were effective at improving visual acuity, with close to half of the eyes improving vision by three lines or more

Samantha Fraser-Bell

samantha.fraser-bell@sydney.edu.au

1 Department of Ophthalmology, University of Sydney, Sydney, NSW, Australia

2 Sydney and Sydney Eye Hospital, Sydney, NSW, Australia from baseline to week 100. Aflibercept was noninferior to ranibizumab at 100 weeks but also not superior. The cheapest of the drugs, compounded bevacizumab, however, was 'not noninferior' to both aflibercept and ranibizumab. Thus, although still an effective treatment, bevacizumab was not considered interchangeable with ranibizumab or aflibercept. At 100 weeks, more eyes treated with aflibercept had central macular thickness within the normal range as measured on optical coherence tomography $(<320 \mu \mathrm{m})$ than in the other groups $(81 \%$ in aflibercept vs $66 \%$ in ranibizumab and $59 \%$ in bevacizumab groups). This was achieved with fewer injections $(n=10.0)$ compared with ranibizumab $(n=11.8)$ or bevacizumab $(n=11.5)$. This difference in number of injections over 2 years was small but may become significant over further years of treatment. Many of our patients with CRVO have multiple comorbidities and competing priorities so fewer treatments can aid compliance and prevent undertreatment, which is more likely to occur outside a clinical trial.

Ranibizumab and aflibercept are heavily subsided in Australia by the federal government for most patients with macular oedema due to CRVO (without thickness restrictions but for eyes with vision between $6 / 12$ and 6/120). Bevacizumab is therefore used less frequently. However, eyes with vision better than $6 / 12$ at initiation of treatment are more likely to receive bevacizumab due to the lack of reimbursement of the licensed medications. We note that eyes with vision of as good as 6/9.5 were included in the LEAVO study.

The label in Australia allows different treatment strategies for managing eyes with CRVO. After stabilisation, macular oedema in these eyes often reliably recurs at a constant interval, which varies between individual eyes. The challenge is finding this interval to prevent under- or overtreatment. It may be that a treat and extend approach is more likely to find this critical recurrence interval, which may be at 6 weeks or 10 weeks rather than 4,8 or 12 weeks, which are the only options in a PRN trial. Recurrence of macular oedema may precede a drop in vision; thus, it might be prudent to treat 
eyes with significant recurrence of oedema even with good vision rather than waiting for the vision to drop.

The LEAVO study has given us useful comparative information about the effect of the three currently available VEGF inhibitors on eyes with CRVO over 2 years. Although effective, bevacizumab was not interchangeable with ranibizumab and aflibercept. Longer studies with different treatment strategies could further inform optimal management of patients with CRVO.

\section{Compliance with ethical standards}

Conflict of interest The authors declare that they have no conflict of interest.
Publisher's note Springer Nature remains neutral with regard to jurisdictional claims in published maps and institutional affiliations.

\section{References}

1. McIntosh RL, Rogers SL, Lim L, Cheung N, Wang JJ, Mitchell P. et al. Natural history of central retinal vein occlusion: an evidence-based systematic review. Ophthalmology. 2010;117: 1113-23.

2. Hykin P, Prevost AT, Vasconcelos JC, Murphy C, Kelly J, et al. LEAVO Study Group. Clinical effectiveness of intravitreal therapy with ranibizumab vs aflibercept vs bevacizumab for macular edema secondary to central retinal vein occlusion: a randomized clinical trial. JAMA Ophthalmol. 2019. https://doi.org/10.1001/jamaophtha lmol.2019.3305. [Epub ahead of print]. 